\title{
MicroRNAs associated to single drug components of R-CHOP identifies diffuse large B-cell lymphoma patients with poor outcome and adds prognostic value to the international prognostic index
}

\author{
Hanne Due ${ }^{1,2+}$, Rasmus Froberg Brøndum ${ }^{1,3+}$, Ken H. Young ${ }^{4}$, Martin Bøgsted ${ }^{1,2,3}$ and Karen Dybkær ${ }^{1,2,3^{*}}$ (D)
}

\begin{abstract}
Background: Treatment resistance is a major clinical challenge of diffuse large B-cell lymphoma (DLBCL) where approximately $40 \%$ of the patients have refractory disease or relapse. Since DLBCL is characterized by great clinical and molecular heterogeneity, the purpose of the present study was to investigate whether miRNAs associated to single drug components of R-CHOP can improve robustness of individual markers and serve as a prognostic classifier.

Methods: Fifteen DLBCL cell lines were tested for sensitivity towards single drug compounds of the standard treatment R-CHOP: rituximab (R), cyclophosphamide $(\mathrm{C})$, doxorubicin $(\mathrm{H})$, and vincristine $(\mathrm{O})$. For each drug, cell lines were ranked using the area under the dose-response curve and grouped as either sensitive, intermediate or resistant. Baseline miRNA expression data were obtained for each cell line in untreated condition, and differential miRNA expression analysis between sensitive and resistant cell lines identified 43 miRNAs associated to growth response after exposure towards single drugs of R-CHOP. Using the Affymetrix HG-U133 platform, expression levels of miRNA precursors were assessed in 701 diagnostic DLBCL biopsies, and miRNA-panel classifiers predicting disease progression were build using multiple Cox regression or random survival forest. Classifiers were validated and ranked by repeated cross-validation.
\end{abstract}

Results: Prognostic accuracies were assessed by Brier Scores and time-varying area under the ROC curves, which revealed better performance of multivariate Cox models compared to random survival forest models. The Cox model including miR-146a, miR-155, miR-21, miR-34a, and miR-23a miR-27a miR-24-2 cluster performed the best and successfully stratified GCB-DLBCL patients into high- and low-risk of disease progression. In addition, combination of the Cox miRNA-panel and IPI substantially increased prognostic performance in GCB classified patients.

Conclusion: As a proof of concept, we found that expression data of drug associated miRNAs display prognostic utility and adding these to IPI improves prognostic stratification of GCB-DLBCL patients treated with R-CHOP.

Keywords: Diffuse large B-cell lymphoma (DLBCL), Drug response, miRNA, Prognosis, Classification

\footnotetext{
* Correspondence: k.dybkaer@rn.dk

${ }^{\dagger}$ Hanne Due and Rasmus Froberg Brøndum contributed equally to this work.

${ }^{1}$ Department of Hematology, Aalborg University Hospital, Sdr. Skovvej 15,

DK-9000 Aalborg, Denmark

${ }^{2}$ Department of Clinical Medicine, Aalborg University, Aalborg, Denmark

Full list of author information is available at the end of the article
}

(c) The Author(s). 2020 Open Access This article is distributed under the terms of the Creative Commons Attribution 4.0 International License (http://creativecommons.org/licenses/by/4.0/), which permits unrestricted use, distribution, and reproduction in any medium, provided you give appropriate credit to the original author(s) and the source, provide a link to the Creative Commons license, and indicate if changes were made. The Creative Commons Public Domain Dedication waiver (http://creativecommons.org/publicdomain/zero/1.0/) applies to the data made available in this article, unless otherwise stated. 


\section{Background}

Diffuse large B-cell lymphoma (DLBCL) is the most common type of malignant lymphoma, accounting for $30-40 \%$ of all newly diagnosed non-Hodgkin lymphomas. It is a highly aggressive and heterogeneous disease with respect to clinical presentation, tumor biology, and prognosis [1]. Gene expression profiling (GEP) enables cell-of-origin classification of DLBCL into two histologically indistinguishable molecular subclasses: the activated B-cell-like (ABC) and the germinal center B-cell-like (GCB), which reflect a subset of the normal B-cell differentiation stages. These subclasses differ in pathogenesis, genetic aberrations, and survival outcome [2, 3] and have entered clinical prognostic evaluation, complementing the international prognostic index (IPI), which has been the gold standard for decades [3, 4].

First-line treatment for newly diagnosed DLBCL patients is a multi-agent regimen combining the anti-CD20 monoclonal antibody rituximab with the three chemotherapeutics cyclophosphamide, doxorubicin, and vincristine, and the corticosteroid prednisone ( $\mathrm{R}-\mathrm{CHOP}$ ). Although addition of rituximab to the regimen has improved treatment outcome of DLBCL substantially, up to $40 \%$ of patients have refractory disease or relapse after initial response to therapy due to drug-specific molecular resistance [5-7]. Study of the pharmacological principles underlying the $\mathrm{R}-\mathrm{CHOP}$ regimen revealed no synergistic interaction but very low cross-resistance, showing a strong combination of independently effective drugs without overlapping mechanisms of resistance [8]. Thus, identification of biomarkers predictive for single drug components of $\mathrm{R}-\mathrm{CHOP}$ is of great importance when attempting to improve clinical outcome.

microRNAs (miRNAs) are endogenous, small noncoding RNA molecules regulating gene expression at the post-transcriptional level [9]. Compelling evidence has demonstrated that miRNA expression is dysregulated in human cancers, with several miRNAs functioning as oncogenes or tumor suppressors [10]. Deregulation of miRNAs occurs early and consistently in tumor development and progression, and thus constitutes a promising source for discovery of novel biomarkers. Indeed, specific miRNAs and global miRNA expression profiles have shown significant potential as diagnostic as well as prognostic biomarkers for DLBCL [11-13], and several studies support their role in chemotherapy resistance [14-16]. Since DLBCL is a highly heterogeneous disease at the molecular level, we hypothesized that a panel of miRNAs associated to individual components of $\mathrm{R}$-CHOP can improve robustness of individual markers and serve as a prognostic classifier predicting disease progression in DLBCL patients.
To test this hypothesis, we determined the global miRNA transcriptome and performed systematic doseresponse drug screens in a DLBCL-specific cell line panel to identify single drug associated miRNAs. Applying multivariate Cox regression and random survival forest techniques, prognostic miRNA-panel classifiers were developed and the predictive accuracies were subsequently evaluated by Brier scores and time varying area under the ROC curves.

\section{Methods \\ Cell lines}

Fifteen human DLBCL-derived cell lines DB, NU-DHL-1, NU-DUL-1, MC-116, SU-DHL-4, SU-DHL-5 (DSMZ, German Collection of Microorganisms and Cell Cultures) and FARAGE, HBL-1, OCI-Ly3, OCI-Ly7, OCI-Ly8, OCILy19, RIVA, SU-DHL-8, and U2932 (Provided by Dr. Jose A. Martinez-Climent, Molecular Oncology Laboratory, University of Navarra, Pamplona, Spain) were included (Table 1). The cell lines were cultured under standard conditions at $37^{\circ} \mathrm{C}$ in humidified atmosphere of 95\% air and $5 \% \mathrm{CO}_{2}$ with RPMI1640 medium containing 10\% fetal bovine serum (FBS) and 1\% penicillin/streptomycin $(\mathrm{P} / \mathrm{S})$ for no longer than 20 passages. All cell lines were authenticated by DNA barcoding as previously described [18] and examined for mycoplasma infection when terminating their culturing period.

\section{Clinical cohorts}

The study was conducted in accordance with the Declaration of Helsinki and tumor biopsies from 73 primary DLBCL patients were collected at time of diagnosis in accordance with the RetroGen research protocol, approved by the Health Ethic Committee of North Denmark Region (Approval jr. no. N-20140099). All patients were treated with $\mathrm{R}-\mathrm{CHOP}$ according to standard protocols. This retrospective cohort is referred to as the AAU dataset. In addition, we used the following data sets from the National Center for Biotechnology Information Gene Expression Omnibus (GEO) repository: Lymphoma/Leukemia Molecular Profiling Project RCHOP (LLMPP R-CHOP) (GSE10846) [19] and International DLBCL Rituximab-CHOP Consortium MD Anderson Project (IDRC) (GSE31312) [20]. All patients were classified into the molecular ABC/GCB subclasses using GEP (Table 2).

\section{Dose-response experiments}

Dose-response screens with rituximab, cyclophosphamide, doxorubicin, and vincristine, respectively, were performed as described previously $[18,21]$. Since cyclophosphamide is a prodrug that requires hepatic activation to produce its active metabolite, the synthetic oxazaphophorine derivate mafosfamide was used in the 
Table 1 Cell line specifications

\begin{tabular}{|c|c|c|c|c|c|c|c|c|c|c|}
\hline & \multirow{2}{*}{$\begin{array}{l}\text { Seeding concentration } \\
\text { cells } / \mathrm{mL}\end{array}$} & \multicolumn{2}{|c|}{ Rituximab } & \multicolumn{2}{|c|}{ Cyclophosphamide } & \multicolumn{2}{|c|}{ Doxorubicin } & \multicolumn{2}{|c|}{ Vincristine } & \multirow[t]{2}{*}{$\mathrm{ABC} / \mathrm{GCE}$} \\
\hline & & AUC & & $A \cup C$ & & AUC & & $\mathrm{AUC}$ & & \\
\hline DB & $0.125 \times 10^{6}$ & 318.1 & $\operatorname{lnt}$ & 345.6 & Res & 275.5 & Res & 130.7 & Res & GCB \\
\hline FARAGE & $0.25 \times 10^{6}$ & 344.1 & Int & 311.0 & Res & 179.6 & Sen & 56.1 & Sen & GCB \\
\hline HBL-1 & $0.25 \times 10^{6}$ & 421.9 & Res & 226.0 & Int & 272.2 & Int & 84.5 & Int & $A B C$ \\
\hline MC-116 & $0.25 \times 10^{6}$ & NA & NA & NA & NA & 271.8 & Int & 62.0 & $N A^{*}$ & GCB \\
\hline NU-DHL-1 & $0.125 \times 10^{6}$ & 316.6 & $\operatorname{lnt}$ & 171.7 & Sen & 200.6 & Sen & NA & NA & $A B C$ \\
\hline NU-DUL-1 & $0.25 \times 10^{6}$ & 236.4 & Sen & 214.1 & Sen & 223.9 & Int & 90.3 & Int & UC \\
\hline OCl-Ly3 & $0.125 \times 10^{6}$ & 407.6 & Res & 261.7 & Int & 253.4 & Int & 74.8 & Int & $A B C$ \\
\hline OCI-Ly7 & $0.125 \times 10^{6}$ & 218.3 & Res & 257.9 & Sen & 324.9 & Res & 114.5 & Int & GCB \\
\hline OCl-Ly8 & $0.25 \times 10^{6}$ & 406.6 & Res & NA & NA & NA & NA & NA & NA & UC \\
\hline OCl-Ly19 & $0.25 \times 10^{6}$ & 400.4 & Res & 202.1 & Sen & 166.9 & Sen & 54.0 & Sen & UC \\
\hline RIVA & $0.25 \times 10^{6}$ & 275.8 & Res & 296.0 & Int & 326.7 & Res & 109.0 & Res & $A B C$ \\
\hline SU-DHL-4 & $0.25 \times 10^{6}$ & 150.4 & Sen & NA & NA & 288.8 & Res & NA & NA & GCB \\
\hline SU-DHL-5 & $0.25 \times 10^{6}$ & 251.2 & Sen & 164.5 & Sen & 201.5 & Sen & 57.9 & Sen & GCB \\
\hline SU-DHL-8 & $0.5 \times 10^{6}$ & 425.0 & Res & 270.6 & Int & 222.0 & Sen & 126.1 & Res & GCB \\
\hline U2932 & $0.4 \times 10^{6}$ & 354.1 & $\operatorname{lnt}$ & 329.8 & Res & 294.8 & Res & 85.3 & Int & GCB \\
\hline
\end{tabular}

For each drug, DLBCL cell lines were ranked according to sensitivity based on area under the dose-response curve (AUC) for rituximab, cyclophosphamide, doxorubicin, and vincristine. Division into tertiles defines: Rituximab, 4 sensitive, 5 intermediate, 5 resistant; Cyclophosphamide, 4 sensitive, 4 intermediate, 4 resistant; Doxorubicin, 5 sensitive, 4 intermediate, 5 resistant; Vincristine, 4 sensitive, 4 intermediate, 4 resistant. Based on GEP, DLBCL cell lines were classified into $\mathrm{ABC} / \mathrm{GCB}$ subclasses by Wright classification using published algorithms at hemaClass.org [17]. ABC, activated B-cell like; GCB, germinal center B-cell like; Int, intermediate; NA, not available; Res, resistant; Sen, sensitive; * excluded due to large variation between replicates

dose-response assays. As the pharmacological effect of $\mathrm{R}-\mathrm{CHOP}$ has limited cross-resistance rather than synergism [8], single drug screens were used instead of combinations in order to identify miRNAs that were specifically associated to response for the individual components of the treatment regimen. The cells were seeded $24 \mathrm{~h}$ prior to addition of the drug using cell line specific seeding concentrations to ensure exponential growth for $48 \mathrm{~h}$ (Table 1). For rituximab dose-response screens, each cell line was subjected to 16 concentrations in serial 2-fold dilutions ranging from $133.3 \mu \mathrm{g} / \mathrm{mL}$ to $407 \times 10^{-5} \mu \mathrm{g} / \mathrm{mL}$ and $30 \mathrm{~min}$ after rituximab addition, normal pooled human $A B$ serum (INR IPLA-SERAB, Novakemi AB, Sweden) was added to a final concentration of $20 \%$. For cyclophosphamide, doxorubicin, and vincristine, the cell lines were exposed to 18 drugspecific concentrations in 2-fold dilutions starting from 80,10 , and $20 \mu \mathrm{g} / \mathrm{mL}$, respectively [21]. The number of metabolic active cells was evaluated after $48 \mathrm{~h}$ of drug exposure using MTS assay (CellTiter 96 Aqueous One Solution Reagent, Promega, Madison, WI). Absorbance was measured at $492 \mathrm{~nm}$ using an Optima Fluorostar plate reader (BMG LAB-TECH, Ortenberg, Germany). All border wells were omitted from data analysis in order to avoid border effect. All drug screens were conducted with 3 replicates of each drug dose and with 3 biological replicates for each cell line. Since a fixed time of drug exposure were used, fast proliferating cells will appear more sensitive compared to slow proliferating ones. Therefore, area under the dose-response curve were used as summary statistic of the drug screens, making the results independent of cell line doubling time as it takes growth kinetic into account [21].

\section{Global miRNA and mRNA expression profiling}

Total RNA was extracted using a modified protocol combining TRIzol Reagent (Invitrogen, Paisley, UK) and mirVana miRNA Isolation Kit (Ambion/ThermoFisher Scientific, Grand Island, NY) as previously described [22]. RNA quality and concentration was determined by Agilent 2100 Bioanalyzer analysis (Agilent Technologies, Santa Clara, CA) and NanoDrop ND-1000 spectrophotometer (ThermoFisher Scientific), respectively. miRNA expression profiling was performed using GeneChip miRNA 1.0.2 arrays (Affymetrix, Santa Clara, CA) according to the manufacturer's protocol. The cell lines DB, FARAGE, OCI-Ly3, OCI-Ly7, OCI-Ly8, OCI-Ly19, NU-DHL-1, RIVA, and U2932 were prepared for hybridization using Flashtag HSR kit from Genesphere (Genesphere, Hatfield, PA) whereas HBL-1, MC-116, NU-DUL-1, SU-DHL-4, SU-DHL-5, and SU-DHL-8 were prepared using Fashtag Biotin HSR RNA labeling kit (Affymetrix) [14]. For GEP, RNA was labeled and hybridized to Affymetrix GeneChip Human Genome U133 (HG-U133) Plus 2.0 arrays, as described by the manufacturer. Generated miRNA and HG-U133 CEL files are 
deposited at NCBI GEO repository GSE72648 and GSE109027, respectively. The data comply with MIAME requirements [23].

\section{Experimental validation of miRNA expression in cell lines by digital droplet polymerase chain reaction}

Two independent cDNA syntheses were conducted and pooled before amplification in digital droplet polymerase chain reaction (ddPCR) analysis. Each sample was analyzed in duplicate/triplicate using ddPCR assays and correlated to probes on $\mathrm{U} 133+2$; hsa-miR-146a (000468), hsa-miR-155 (002623), hsamiR-21 (000397), hsa-miR-27a (000408) and hsa-miR34a (000426). miRNA expression was normalized to RNU6B (001093) and RNU24 (001001) and $\log 2$ transformed prior to correlation to miRNA specific probes on U133 + 2 (232504_at for miR-146a, 22937 _ at for miR-155, 220990_s_at for miR-21, 1555847_a at for miR-23a -miR-27a-miR-24-2, and 235571_at for miR-34a). Correlation coefficients shown in Supplementary Figure 5.

\section{Statistical analysis}

All statistical analyses were performed with $\mathrm{R}$ version 3.5.1; an accompanying knitR document with detailed information on the analysis and package versions is supplied in Supplementary Document S2. Prior to statistical analysis, the array data were cohort-wise background corrected and normalized at probe level by robust multichip average (RMA) [24] implemented in the Bioconductor package affy v1.58 [25].

Differentially expressed miRNAs between DLBCL cells lines classified as sensitive and resistant for rituximab, cyclophosphamide, doxorubicin, and vincristine, respectively, were identified using the empirical Bayes method [26] implemented in the $\mathrm{R}$ package limma v3.36.3 [27]. Since cell lines were prepared for hybridization to the miRNA 1.0.2 microarray platform with different labeling kits [14], differential miRNA expression analyses were adjusted for possible confounding with a kit effect by including it as a covariate in the model. MiRNAs with absolute log-fold changes greater than two $(|\mathrm{FC}|>2)$ were considered as differentially expressed and were included in the list of candidate miRNAs subjected for further analysis. The set of candidate miRNAs for development of the prognostic classifier was chosen by filtering the set of differentially expressed miRNAs against those detected by HG-U133 probe sets. For each candidate probe set, correlation analysis between miRNA 1.0.2 array and HG-U133 array was conducted to validate HG-U133 array-based miRNA expression assessment.

Three clinical data sets (Table 2) were combined into a meta-cohort for training and validation of the prognostic classifiers. Validation was performed by
Table 2 Patient characteristics

\begin{tabular}{llll}
\hline & IDRC & LLMPP R-CHOP & AAU \\
\hline Gender & 468 & 233 & 73 \\
$\quad$ Female & $198(42 \%)$ & $99(42 \%)$ & $30(41 \%)$ \\
$\quad$ Male & $270(58 \%)$ & $134(58 \%)$ & $43(59 \%)$ \\
Age & & & \\
Median & 63 & 61 & 66 \\
Range & $18-92$ & $17-92$ & $20-87$ \\
IPI & & & $48(66 \%)$ \\
0-1 & $254(54 \%)$ & $94(40 \%)$ & $21(29 \%)$ \\
$2-5$ & $168(36 \%)$ & $70(30 \%)$ & $4(5 \%)$ \\
NA & $46(10 \%)$ & $69(30 \%)$ & $32(44 \%)$ \\
ABC / GCB & & & $32(44 \%)$ \\
ABC & $199(43 \%)$ & $93(40 \%)$ & $9(12 \%)$ \\
GCB & $225(48 \%)$ & $107(46 \%)$ & $33(14 \%)$ \\
UC & $44(9 \%)$ & &
\end{tabular}

Number of patients and percentage within cohort / variable. $A B C$ activated $B-$ cell-like, GCB germinal center B-cell-like, IPI International prognostic index, NA not available, $n$ number of patients, UC unclassified

repeated cross-validation with 10 folds and 10 repeats rather than using an independent validation set, since only two large clinical cohorts were available and we wanted to investigate the potential of a model trained on a combined dataset. By repeating the crossvalidation we were able to investigate the variation in prediction accuracy resulting from the randomization in the cross-validation folds.

To compensate for cohort-wise technical batch effects, the ComBat function implemented in the R-package sva [28] was applied. Training of prognostic classifiers were performed for all DLBCL patients and for subsets of $\mathrm{ABC}$ and GCB classified patients, respectively. Progression-free survival (PFS) was chosen as the outcome, since it is a treatment evaluation parameter as closely as possible to the time of drug exposure and the tested miRNAs were all associated directly to drug specific response. Furthermore, overall survival (OS) was used for verification of findings.

The prognostic miRNA-panel models were identified and trained by both multivariate Cox regression or random survival forest with 1000 trees using either drugspecific probes alone or in combination with a dichotomized IPI score (IPI " $0-1$ " and " $2-5$ " for low and high risk, respectively). The random survival forest implementation from the R-package randomForestSRC v2.7.0 was used [29-31]. For Cox regression models, variable selection was performed by preselecting probes that had a statistically significant effect in univariate Cox regressions adjusted for study effects $(p<0.05)$. The preselection of probes was performed for each cross-validation 
fold and repetition to avoid sharing information between training and validation sets. Additionally, univariate Cox regression models using either IPI or age as input features were trained and used as a baseline comparison for the models including miRNA probes. The prognostic accuracies of the classifiers were validated by performing 10-fold cross-validation using either all DLBCL patients in the combined dataset, or the respective subsets of ABC or GCB classified patients. The models were ranked by the Brier score and time varying area under the ROC curves by comparing the predicted survival probability for each individual in the combined validation set from the cross-validations to the observed PFS and OS every half year from zero to five years.

The linear predictions from the Cox models in the cross-validation were used to get a score for each individual, by averaging across the scores from the 10 repeats of the cross-validation. By splitting these scores into tertiles Kaplan-Meier plots for low, intermediate, and high risk patients were generated within the respective cohorts and with significance evaluated by log-rank tests. Since more than $90 \%$ of events for both PFS and OS happened within the first 5 years Kaplan-Meier plots were restricted to this period. For all Kaplan-Meier analyses, significance threshold were set to 0.05 .

\section{Results}

\section{Identification of miRNAs associated with drug-specific response}

Triplicate dose-response experiments were analyzed using area under the dose-response curve for rituximab, cyclophosphamide, doxorubicin, and vincristine, respectively, taking the individual cell line doubling time into account [21]. For each drug, the cell lines were ranked according to their sensitivity, grouped into tertiles and categorized as sensitive, intermediate responsive, or resistant (Table 1). Global miRNA expression screens were conducted on untreated cell lines for optimal candidate selection and subsequent differential miRNA expression analysis between sensitive and resistant cell lines identified 43 miRNAs to be associated with compounds of the R-CHOP regimen (Supplementary Tables 1, 2, 3, and 4). The majority of miRNAs associated with vincristine and doxorubicin were downregulated in resistant DLBCL cells, whereas rituximab resistance was primarily associated with upregulated miRNAs (Supplementary Tables 1, 3, and 4). Conversely, differentially expressed miRNAs for cyclophosphamide were equally distributed between up- and downregulation (Supplementary Table 2). Several miRNAs were associated with more than one drug (Fig. 1); of notice, 6 out of 13 miRNAs were shared between vincristine and doxorubicin. Additionally, miR-146a, miR-148a, miR-155, miR-221, and

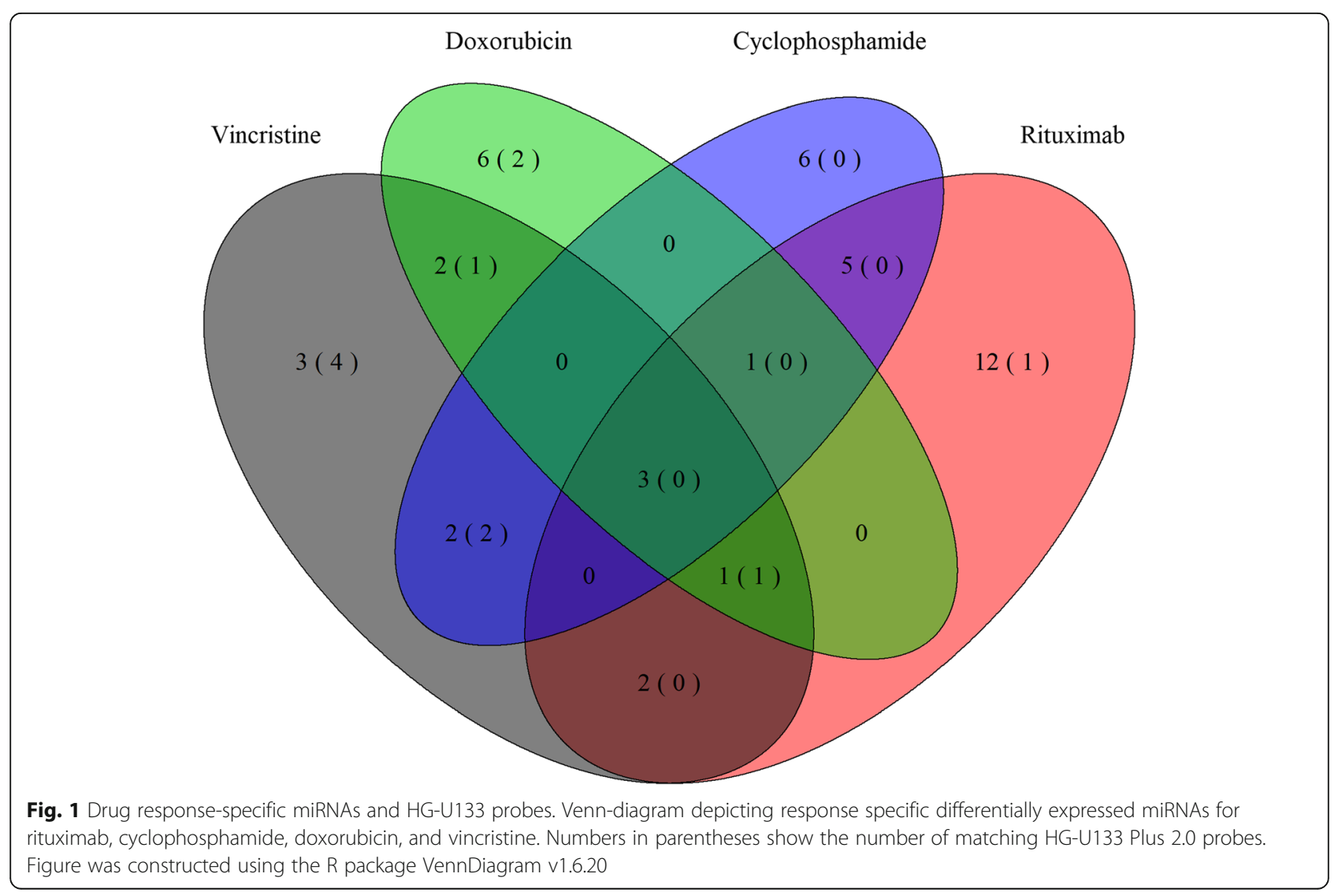


miR-222 displayed ambiguous association to responses of different compounds of the regimen.

Since the miRNA microarray platform is not used in clinical context, miRNAs were matched to HG-U133 microarray probe sets detecting miRNA encoding genes, which reduced the candidate list to 11 probe sets detecting the following 9 miRNAs: miR-146a, miR-155, miR21, miR-23a 27a 24-2 cluster, miR-34a, miR-503, and let-7b, which cover drug specific miRNAs for all four drugs (Fig. 1, Table 3).

\section{miRNA-panel prognostic classifier}

Based on the identified drug-specific miRNAs, multiple Cox regression and random survival forest models were used to build classifiers predicting disease progression in patients with DLBCL to test both parametric and nonparametric ensemble based survival models. Since ABC and GCB-classified patients display different miRNA expression patterns, pathogenesis and clinical outcome [2, $3,11]$, the training and validation of prognostic models were conducted for all DLBCL patients and for $A B C$ and GCB classified patients, respectively. Additionally, since IPI is well-established in the clinical setting, it was essential to evaluate if the prognostic miRNA classifiers added to the prognostic performance of IPI.

Prognostic accuracies of the generated miRNA-panel classifiers were assessed by Brier scores, which revealed better performance of the multivariate Cox models compared to the random survival forest models that had the largest prediction error regardless of input features (Fig. 2a-c). In contrast, evaluations of predictive performances by time varying area under the ROC curves were not as unambiguous, however, the highest predictive accuracy was still observed for multivariate Cox models (Fig. 2d-e). Comparison of analyses conducted for the respective cohorts (All DLBCL, ABC, and GCB patients) showed the lowest prediction errors for all models within the GCB subclass (Fig. 2a-c), with a multivariate Cox miRNA-panel model displaying prognostic utility comparable to IPI (Fig. 2f). In addition, combination of the miRNA-panel and IPI substantially increased prognostic performance in GCB classified patients (Fig. 2f), indicating a prognostic signal from the response-specific miRNAs independent of IPI. Furthermore, the Cox model combining IPI and the miRNA probe sets within the GCB subclass had the highest prognostic utility for all cross-validation repetitions showing a robust improvement.

In the ABC subclass, the developed miRNA-panel classifier did not provide additional prognostic information to IPI, either alone or in combination with IPI (Fig. $2 b$ and e), suggesting little utility of the drug-specific miRNAs in this subclass. In agreement, similar results for $\mathrm{ABC}$ and GCB classified patients were observed when comparing the predicted PFS to the observed OS (Supplementary Figure 1). Consequently, the analyses were focused on the GCB subclass of DLBCL, which accounts for $46 \%$ of all cases (Table 2).

A linear prediction score was obtained for each individual in the combined GCB dataset by averaging the scores from the validation sets across the 10 repetitions of cross-validation for the multivariate Cox models using miRNA probe sets alone or in combination with IPI. These scores were used to rank the individual risk for all GCB classified patients, and by splitting the scores into tertiles, all GCB DLBCL patients were classified in

Table 3 Candidate miRNAs

\begin{tabular}{llll}
\hline Probe ID (HG-U133) & miRNA & Drug sensitivity & Annotation grade \\
\hline 232504_at & miR-146a & $\downarrow$ Cyc, $\uparrow$ Vin & A \\
238225_at & miR-146a & $\downarrow$ Cyc, $\uparrow$ Vin & B \\
229437_at & miR-155 & $\downarrow$ Rtx, $\uparrow$ Dox, $\uparrow$ Vin & A \\
220990_s_at & miR-21 & $\uparrow$ Vin & A \\
229417_at & miR-21 & $\uparrow$ Vin & E \\
235317_at & miR-23a miR-27a miR-24-2 & $\uparrow$ Dox, $\uparrow$ Vin & B \\
1555847_a_at & miR-23a miR-27a miR-24-2 & A Dox, $\uparrow$ Vin & A \\
235571_at & miR-34a & $\uparrow$ Dox, $\uparrow$ Vin & A \\
1557342_a_at & let-7b & $\uparrow$ Vin & B \\
241464_s_at & let-7b & $\uparrow$ Vin & B \\
227488_at & miR-503 & $\downarrow$ Rtx &
\end{tabular}

Differentially expressed miRNAs detected by HG-U133 Plus 2.0 probes were selected as candidate miRNAs. The list consisted of 11 probe sets detecting 9 miRNAs. The annotation grade was assessed by NetAffx (Affymetrix) a transcript assignment pipeline creating a relationship between GeneChip probe sets and current transcript record. The transcript assignment grades fall into five categories A-E that describe the quality of the direct evidence. Grade $A$ is a matching probe set having nine or more probes matching transcript mRNA. Grade B transcript assignments have partial overlap between transcripts and target sequence. Grade $E$ is given when no transcript is found. Abbreviations: $\uparrow$ and $\downarrow$ defines up- and downregulation, respectively, in drug sensitive cell lines. Cyc, cyclophosphamide; Dox, doxorubicin; Rtx, rituximab; Vin, vincristine 

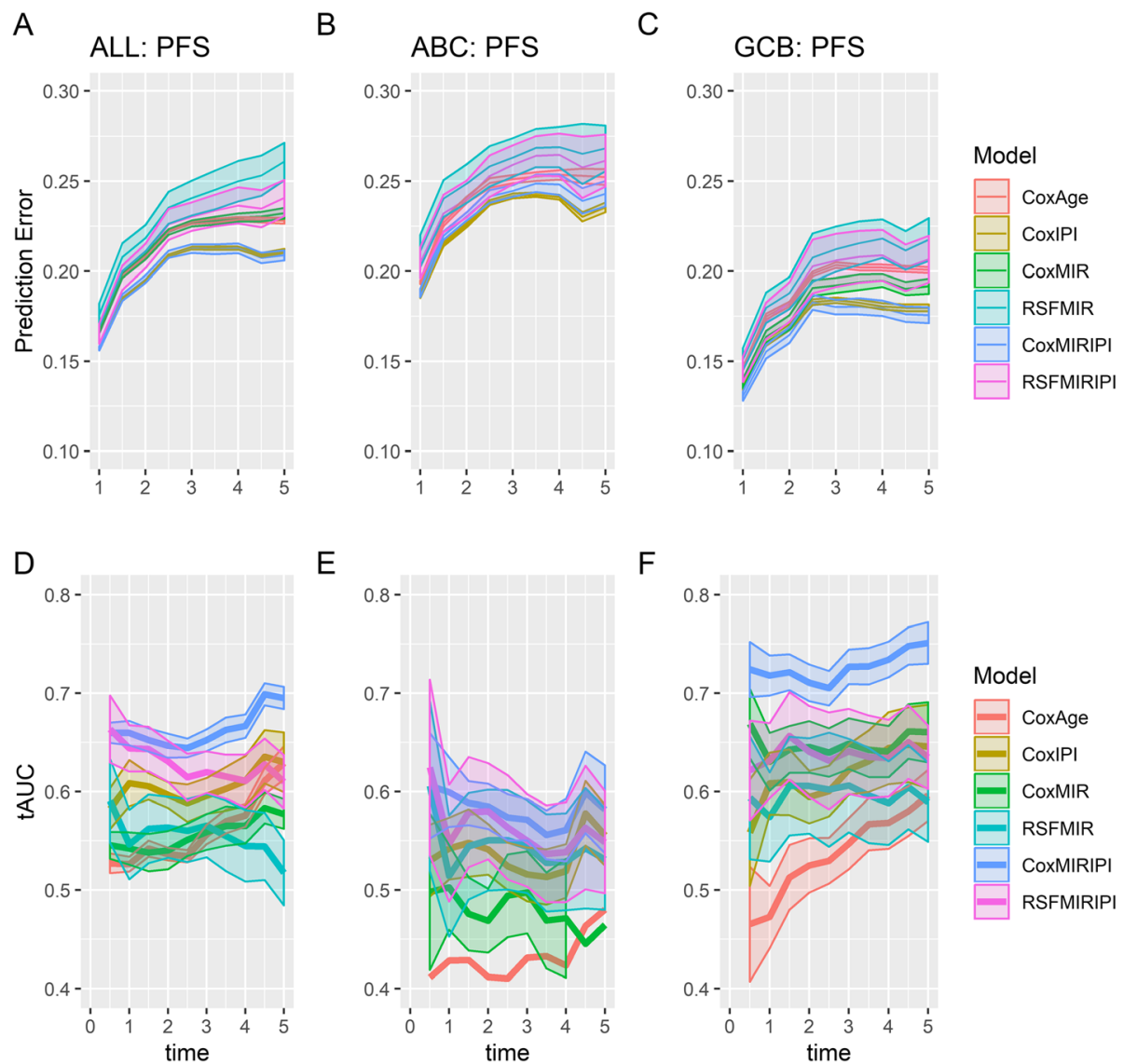

$\mathrm{E}$

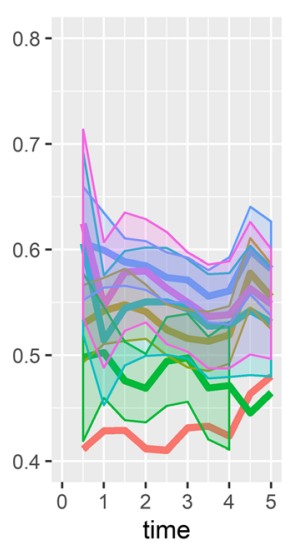

$\mathrm{F}$

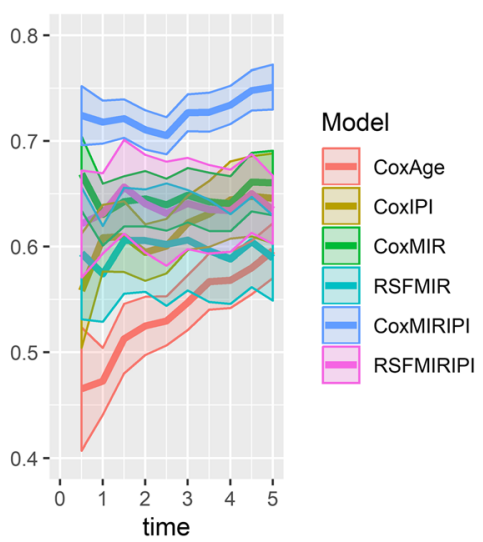

Fig. 2 Evaluation of prognostic accuracy. Predicted survival from various prognostic classifiers vs observed progression free survival with the Brier score (top row, a-c) or tAUC (bottom row, $\mathbf{d}-\mathbf{f}$ ). Figures display means +-2SD evaluated across the 10 cross-validation repetitions. The prognostic classifiers include: multivariate Cox regression models using either age (CoxAge), IPI (CoxIPI), miRNA expression (CoxMIR), or miRNA expression combined with IPI score (CoxMIRIPI), and random survival forest models using miRNA expression (RSFMIR) or miRNA expression in combination with IPI (RSFMIRIPI). Time in years. Figure was constructed using the R packages ggplot2 v3.2.1 and gridExtra v2.3. ABC, activated B-cell-like; GCB, germinal center B-cell-like; IPI, international prognostic index; MIR, microRNA panel; PFS, progression-free survival; RSF, random survival forest; tAUC, time-varying are under the ROC curve

defined groups of low, intermediate, and high-risk, the latter with significantly inferior prognosis as shown in the Kaplan-Meier plots for PFS and OS (Fig. 3, Supplementary Figure 2). The low and intermediate-risk group are not very distinct for the model including only miRNA probe sets, addition of IPI, however, clearly separates the patients into the distinct risk groups. The prognostic potential of the miRNA-panel was tested in each individual dataset validating the findings of inferior survival of patients with high-risk score (Supplementary Figure 3a-d).

In accordance, GCB DLBCL patients with stable or progressive disease at time of response evaluation [32] display higher risk scores (Fig. 4), with the biggest difference in mean risk scores for the model including IPI. In addition, the multivariate Cox regression model combining IPI and probe sets detecting drug-specific miRNAs (miR-146a, miR-155, miR-21, miR-34a, and the miR- 23a miR-27a miR-24-2 cluster) displayed the strongest prognostic performance (Fig. $2 \mathrm{c}$ and $\mathrm{f}$ ) and was selected as the best model. Selected features and coefficients of the developed prognostic classifier are presented in Table 4, showing that most of the prognostic signal is carried by IPI, and that some of the probes have insignificant signal, although they were significant in the univariate analysis (Supplementary Table 5). Six of the candidate probe sets were not included in the final model, as they did not display significant effect in univariate Cox regression analysis (Supplementary Table 5).

Expression levels of the probe sets included in the final prognostic model (Table 4) were highly correlated to the mature miRNA measured by miRNA array (Supplementary Figure 4), supporting HG-U133 array-based miRNA expression assessment. Of notice, expression of the six miRNAs without significant effect were not correlated to the mature miRNA 
A

\section{MIR: PFS}

Strata + Low + Intermediate + High

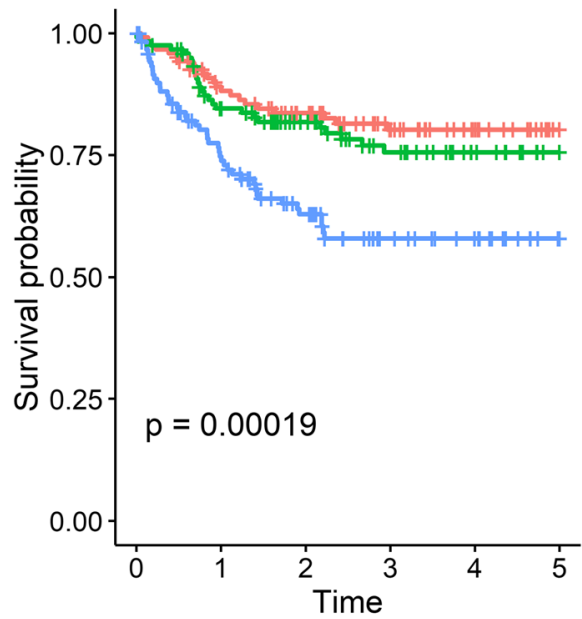

A

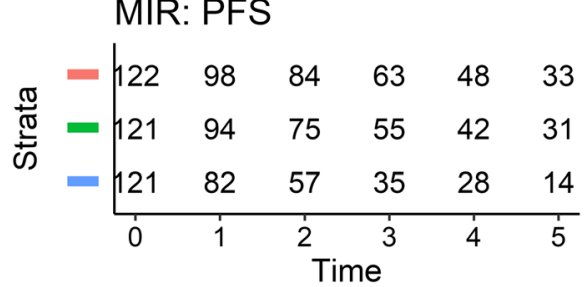

B

\section{MIR+IPI: PFS}

Strata + Low + Intermediate + High

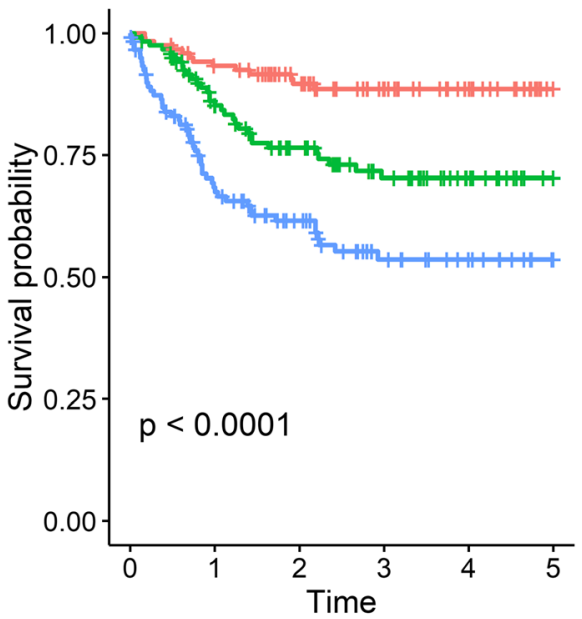

B

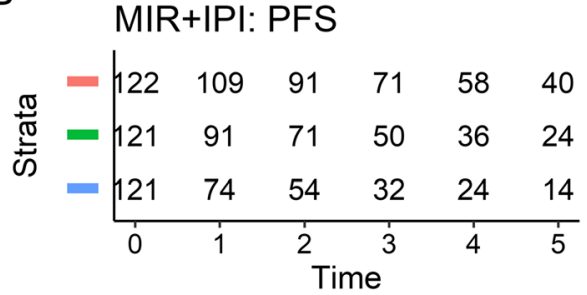

Fig. 3 Predicted risk group vs 5-year progression free survival in the combined GCB dataset. Risk groups were obtained by taking the average predicted risk score across validation folds for the repeated cross-validation for the multivariate Cox models using (a) miRNA probes either alone or (b) in combination with IPI and splitting these into tertiles. Figure was constructed using the R package survminer v0.4.6

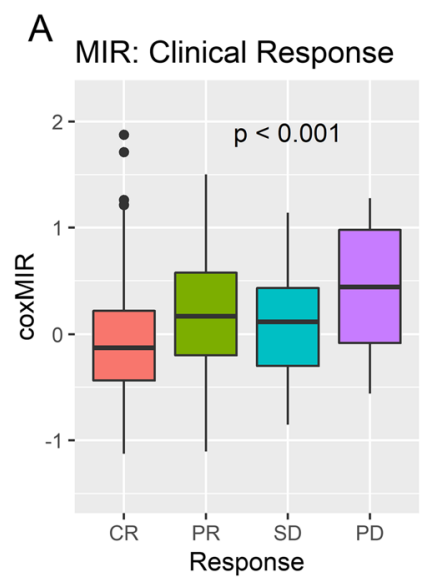

B MIR+IPI: Clinical Response

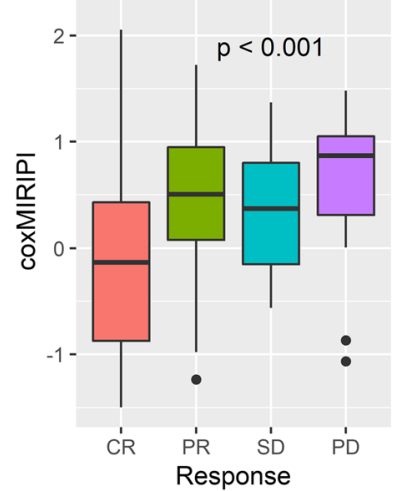

Response

官 Complete Response $(\mathrm{N}=248)$

Partial Response $(\mathrm{N}=48)$

Stable Disease $(\mathrm{N}=12)$

Progressive Disease $(\mathrm{N}=17)$

Fig. 4 Risk scores in Cheson response evaluation classes. Response evaluations of GCB classified patients were investigated for association to predicted risk scores. CR, complete remission; PD, progressive disease; PR, partial response; SD, stable disease. Risk scores were obtained by taking the average predicted risk score across validation folds for the repeated cross-validation for the multivariate Cox models using miRNA probes either alone or in combination with IPI. Figure was constructed using the R packages ggplot2 v3.2.1 and gridExtra v2.3 
Table 4 Selected features and corresponding coefficients for the final multivariate Cox model

\begin{tabular}{lll}
\hline Feature & miRNA & Hazard Ratio \\
\hline IPI2-5 & & 3.335 \\
232504_at & miR-146a & 0.857 \\
229437_at & miR-155 & 0.868 \\
220990_s_at & miR-21 & 0.995 \\
1555847_a_at & miR-23a miR-27a miR-24-2 & 0.958 \\
235571_at & miR-34a & 0.776
\end{tabular}

The model was trained within the GCB subset of patients. MiRNA probes were pre-filtered to only include those significant in univariate Cox regression

(Supplementary Figure 4), most likely due to low probe set specificity (Annotation Grade B and E, Table 3). For all drug-specific miRNAs a hazard ratio below 1 is observed (Table 4), indicating that higher expression is associated with better prognosis corresponding to the original observation of downregulation being associated to resistance in vitro (Table 3). Additionally, it is evident that vincristine and doxorubicin are the main contributors to the prognostic miRNA signature as most of the miRNAs in the final classifier were initially included due to significant effect on doxorubicin and vincristine resistance.

\section{Discussion}

Here, we combined global miRNA expression profiles and systematic dose-response screens to identify miRNAs associated to growth responses towards single drug components of the R-CHOP regimen. MiRNA-panel classifiers that assign individual DLBCL patients into low- and high-risk patients were generated utilizing two different approaches, multivariate Cox regression and random survival forest.

Several of the identified drug-specific miRNAs displayed ambiguous association to compounds of the R$\mathrm{CHOP}$ regimen. Thus, high expression of miR-155 was associated with sensitivity to both doxorubicin and vincristine and with resistance to rituximab, most likely due to different drug mechanisms of actions and affected target genes. Noteworthy, doxorubicin and vincristine had $46 \%$ of the response specific miRNAs in common (miR23a miR-27a miR-24, miR-34a, miR-155, and miR222), indicating resistance mechanisms affecting the same molecular pathways. Consistently, ectopic induction of miR-34a have been shown to sensitize Ewing's sarcoma cells to doxorubicin and vincristine [33].

Anti-tumor drugs often have several mechanisms of actions. Cyclophosphamide has, beside the function as an alkylating agent, been shown to induce cytokine release from the cancer cells, thereby attracting macrophages and facilitating antibody-mediated elimination
[34]. Our in vitro drug screen system lacks stromal cells and the tumor microenvironment, thus, it is important to emphasize that the identified cyclophosphamidespecific miRNAs is restricted to the DNA crosslinking mechanism of action. Furthermore, prednisone is a synthetic corticosteroid drug suppressing the immune system without cytotoxic effects and consequently, not included in this study. The majority of the miRNAs included in the final prognostic miRNA signature are associated with doxorubicin and vincristine, which consistently, are the two major cornerstones for efficacy of R-CHOP [35].

Evaluation of prognostic impact in this study is based on retrospective clinical cohorts where treatment conditions are not strictly unified regarding number of cycles, dosing $[19,20]$ and individual dose reductions due to side-effects like neuropathy [36]. Taking this and the pharmacological information on no synergistic effect of R-CHOP into account [8], single drug screens were used rather than combinational assays to identify candidate miRNAs to be assessed in non-weighted models using either multivariate Cox regression or random survival forest models. The candidate miRNA originating from single drug screens are thus tested and evaluated for prognostic impacts in DLBCL patients receiving the full R-CHOP regimen, ensuring that only miRNAs with enough statistical power alone in univariate Coxregression analysis and in modelled combinations are included in the final miRNA signature/panel.

The gene expression-based $\mathrm{ABC} / \mathrm{GCB}$ subclasses of DLBCL rely on distinct oncogenic mechanisms $[37,38]$ and since affected miRNA targets vary depending upon the cell type and differentiation stage in which the miRNA is expressed [39], prognostic classifiers were generated separately in $\mathrm{ABC}$ and GCB subclasses. The drug-response specific miRNA-panel demonstrated a significant prognostic association with PFS and OS in GCB classified DLBCL patients treated with R-CHOP. Consistently, the complete response rate after $\mathrm{R}-\mathrm{CHOP}$ was significantly higher for low-risk patients than highrisk, documenting association between miRNA expression and response to R-CHOP.

Of the seven miRNAs significant in univariate Cox regression, expression levels of miR-21, miR-146a, and miR-155 have been confirmed to differentiate the $\mathrm{ABC} /$ GCB subclasses in $\geq 4$ studies [11, 12, 40-46], emphasizing the molecular heterogeneity between the two molecular subclasses of DLBCL. This difference in expression levels as well as the cell type specific effect of a particular miRNA, could be the reason why only little prognostic utility of the miRNA-panel was observed for $A B C$ classified patients in contrast to GCB patients. Additionally, IPI displayed lower prognostic accuracy in these patients compared to GCB classified patients, 
indicating that new prognostic tools for $\mathrm{ABC}-\mathrm{DLBCL}$ are needed.

The prognostic gold standard IPI is based solely on clinical parameters [4] and does not provide insight into the molecular pathways driving tumorigenesis and treatment resistance. Thus, molecular markers could potentially improve the prognostic accuracy of IPI and simultaneously provide information about the underlying molecular mechanisms. In line, combination of IPI with drug-specific miRNAs increased the prognostic accuracy in GCB-DLBCL. miRNAs display potential as a promising source of biomarkers as they, due to their small size, are relatively resistant to RNase degradation and are well-preserved in FFPE tissue [47]. Additionally, miRNAs can be detected in plasma and serum, thus, holding potential as liquid biomarkers $[45,48]$. However, this study is proof of concept that addition of single drug-specific miRNAs to IPI improves prognostic stratification.

The prognostic utility of the miRNA probe sets was tested by training models using both the widely used semi-parametric multivariate Cox model, and the non-parametric ensemble based random survival forest. The Cox models relies on an assumption of proportional hazards and has a straightforward interpretation of parameters, whereas the random forest model makes no such assumptions and is able to fit more complex interactions among variables in the training data, but is more difficult to interpret. However, in our data we found no benefit of the random survival model compared to the Cox model. This might be caused by the limited number of variables which means that the individual survival trees are of limited depth, leading to too much bias in the predictions. Experimental validation of miRNA expression levels in cell lines are performed by ddPCR prior to correlation to miRNA specific probes on $\mathrm{U} 133+2$ (Supplementary Figure 4) generating coefficients of correlations ranging between 0.65 and 0.94 (Supplementary document S2, Table 5) supporting the usability of miRNAs in prognostic models with IPI.

A possible drawback of the current study is the lack of an independent external validation set, since the prediction accuracies from the cross-validation might be overestimated. The focus of the current study is, however, not the absolute but the relative prediction accuracy and thus ranking of different models. The ranking of the models could be affected by the randomization into crossvalidation folds, results however showed that the multivariate cox model including both miRNA probe sets and IPI had superior prognostic signal within the GCB subgroup, regardless of cross-validation randomization, strengthening the hypothesis that miRNAs carry independent prognostic signal from IPI. However, further studies including an external validation set are needed before making recommendations on clinical application.

The miRNAs included in the final model are well studied miRNAs with known functions in normal B-cell differentiation and tumorigenesis [13, 49, 50]. miR-21 and miR-155 has been reported to be upregulated and to possess oncogenic properties in numerous cancers including breast cancer, glioblastoma, and DLBCL [5155]. In addition, they have shown specific importance in the pathogenesis of DLBCL, highlighted by the fact that transgenic mice overexpressing miR-21 or miR-155 spontaneously develop lymphoma $[56,57]$. In line, high expression of miR-21 is associated with inferior prognosis in DLBCL patients [55], and functional studies document a direct link between high miR-21 expression and CHOP resistance through regulation of PTEN [58]. miR155 has been shown to control vincristine sensitivity in DLBCL cells through downregulation of Wee1 and clinical outcome analysis documented a significantly prolonged survival of GCB-classified DLBCL patient with high miR-155 expression [16].

\section{Conclusions}

In conclusion, we found as proof of concept that adding gene expression data detecting drug-specific miRNAs to the clinically established IPI improved the prognostic stratification of GCB-DLBCL patients treated with RCHOP.

\section{Supplementary information}

Supplementary information accompanies this paper at https://doi.org/10. 1186/s12885-020-6643-8.

Additional file 1: Table 1. Rituximab response-specific miRNAs. Table 2. Cyclophosphamide response-specific miRNAs. Table 3. Doxorubicin response-specific miRNAs. Table 4. Vincristine response-specific miRNAs. Table 5. Uni and multivariate cox regression analysis. Figure 1. Predicted survival from various prognostic classifiers vs observed overall survival with the brier score (top row) or time varying AUC (bottom row). Figure 2. Predicted risk group vs 5 -year overall survival in the combined GCB dataset. Figure 3. Predicted risk group vs 5-year overall survival in GCB classified patients within each dataset: IDRC, LLMPPR-CHOP, and $A A U$. Figure 4. Correlation analysis between mature miRNA and miRNA encoding gene. Figrue 5. Correlation analysis between mature miRNA and miRNA encoding gene.

Additional file 2. MicroRNA associated to single drug components of RCHOP identifies diffuse large B-cell lymphoma patients with poor outcome and adds prognostic value to the international prognostic index.

\section{Abbreviations}

$A B C$ : Activated B-cell-like; ddPCR: Digital droplet polymerase chain reaction; DLBCL: Diffuse large B-cell lymphoma; GCB: The germinal center B-cell-like; GEO: Gene Expression Omnibus; GEP: Gene expression profiling; IDRC: International DLBCL Rituximab-CHOP Consortium MD Anderson Project; IPI: International prognostic index; LLMPP R-CHOP: Lymphoma/Leukemia Molecular Profiling Project R-CHOP; miRNA: MicroRNA; OS: Overall survival; PFS: Progression-free survival; R-CHOP: Rituximab, cyclophosphamide, doxorubicin, vincristine, prednisone; RMA: Robust multichip average; tAUC: Time varying area under the ROC curves 


\section{Acknowledgments}

This work was presented at the 61st ASH Annual Meeting \& Exposition, Orlando, December 2019 [59]. We greatly appreciate the technical assistance from Louise Hvilshøj Madsen and Helle Høholt, Department of Haematology, Aalborg University Hospital, Denmark.

\section{Authors' contributions}

H.D, R.F.B, M.B, and K.D designed the research; H.D, R.F.B, K.H.Y, M.B, and K.D collected the data; H.D and R.F.B performed experiments and statistical modelling, H.D and R.F.B. wrote the paper. All authors contributed to data interpretation and critically revised and approved the final manuscript.

\section{Funding}

This work was supported by grants from The Danish Cancer Society and The North Region Research Foundation, Denmark. The role of this grant was to financially support a PhD study of chemotherapy resistance in DLBCL. The funding bodies had no implications on the study design, collections, analysis, and interpretation of data or writing the manuscript.

\section{Availability of data and materials}

The datasets supporting the conclusions of this article are available in the National Center for Biotechnology Information Gene Expression Omnibus (GEO) repository, GSE72648 (https://www.ncbi.nlm.nih.gov/geo/query/acc. cgi?acc=GSE72648) and GSE109027 (https:/www.ncbi.nlm.nih.gov/geo/ query/acc.cgi?acc=GSE109027). In addition, we used the following online available data sets: Lymphoma/Leukemia Molecular Profiling Project R-CHOP (GSE10846) and International DLBCL Rituximab-CHOP Consortium MD Anderson Project (GSE31312).

\section{Ethics approval and consent to participate}

Tumor biopsies were collected in accordance with the RetroGen research protocol, reviewed approved by the Health Ethic Committee of North Denmark Region (Approval jr. no. N-20140099) allowing exemption from the requirement of informed consent according to Sections 3 to 5 in Danish Act on Research Ethics Review of Health Research Projects. Informed consent was waived since this notifiable database research project did not involve any health risks and under the given conditions could not otherwise put a strain on the trial subject.

\section{Consent for publication}

Not applicable.

\section{Competing interests}

The authors declare that they have no competing interests.

\section{Author details}

'Department of Hematology, Aalborg University Hospital, Sdr. Skovvej 15, DK-9000 Aalborg, Denmark. ${ }^{2}$ Department of Clinical Medicine, Aalborg University, Aalborg, Denmark. ${ }^{3}$ Clinical Cancer Research Center, Aalborg University Hospital, Aalborg, Denmark. ${ }^{4}$ Duke University Medical Center, Division of Hematopathology and Department of Pathology, Durham, NC, USA.

\section{Received: 25 September 2019 Accepted: 17 February 2020}

\section{Published online: 20 March 2020}

\section{References}

1. Nogai H, Dörken B, Lenz G. Pathogenesis of non-Hodgkin's lymphoma. J Clin Oncol. 2011;29:1803-11. https://doi.org/10.1200/JCO.2010.33.3252.

2. Alizadeh AA, Eisen MB, Davis RE, Ma C, Lossos IS, Rosenwald A, et al. Distinct types of diffuse large B-cell lymphoma identified by gene expression profiling. Nature. 2000;403:503-11. https://doi.org/10.1038/35000501.

3. Swerdlow SH, Campo E, Pileri SA, Harris NL, Stein H, Siebert R, et al. The 2016 revision of the World Health Organization classification of lymphoid neoplasms. Blood. 2016;127:2375-90.

4. Project TIN-HLPF. A predictive model for aggressive non-Hodgkin's lymphoma. N Engl J Med. 1993;329:987-94. https://doi.org/10.1056/ NEJM199309303291402.

5. Coiffier B, Lepage E, Briere J, Herbrecht R, Tilly H, Bouabdallah R, et al. CHOP chemotherapy plus rituximab compared with $\mathrm{CHOP}$ alone in elderly patients with diffuse large-B-cell lymphoma. N Engl J Med. 2002;346:235-42. https://doi.org/10.1056/NEJMoa011795.

6. Sehn LH, Donaldson J, Chhanabhai M, Fitzgerald C, Gill K, Klasa R, et al. Introduction of combined CHOP plus rituximab therapy dramatically improved outcome of diffuse large B-cell lymphoma in British Columbia. J Clin Oncol. 2005;23:5027-33. https://doi.org/10.1200/JCO.2005.09.137.

7. Friedberg JW. Relapsed/refractory diffuse large B-cell lymphoma. Hematol Am Soc Hematol Educ Progr. 2011;2011:498-505. https://doi.org/10.1182/ asheducation-2011.1.498.

8. Palmer AC, Chidley C, Sorger PK. A curative combination cancer therapy achieves high fractional cell killing through low cross-resistance and drug additivity. Elife. 2019;8:e50036. https://doi.org/10.7554/eLife.50036.

9. Bartel DP. MicroRNAs: genomics, biogenesis, mechanism, and function. Cell. 2004;116:281-97. https://doi.org/10.1016/S0092-8674(04)00045-5.

10. Zhang B, Pan X, Cobb GP, Anderson TA. microRNAs as oncogenes and tumor suppressors. Dev Biol. 2007;302:1-12. https://doi.org/10.1016/J.YDBIO.2006.08.028.

11. labal J, Shen Y, Huang X, Liu Y, Wake L, Liu C, et al. Global microRNA expression profiling uncovers molecular markers for classification and prognosis in aggressive B-cell lymphoma. Blood. 2015;125:1137-45. https://doi.org/10.1182/ blood-2014-04-566778.

12. Due H, Svendsen $P$, Bødker JS, Schmitz A, Bøgsted M, Johnsen HE, et al. miR-155 as a Biomarker in B-Cell Malignancies. Biomed Res Int. 2016;2016:114. doi:https://doi.org/10.1155/2016/9513037.

13. Marques SC, Laursen MB, Bødker JS, Kjeldsen MK, Falgreen S, Schmitz A, et al. MicroRNAs in B-cells: from normal differentiation to treatment of malignancies. Oncotarget. 2015;6:7-25. https:/doi.org/10.18632/oncotarget.3057.

14. Marques SC, Ranjbar B, Laursen MB, Falgreen S, Bilgrau AE, Bødker JS, et al. High miR-34a expression improves response to doxorubicin in diffuse large B-cell lymphoma. Exp Hematol. 2016;44:238-246.e2.

15. Rasmussen MH, Lyskjær I, Jersie-Christensen RR, Tarpgaard LS, PrimdalBengtson B, Nielsen MM, et al. miR-625-3p regulates oxaliplatin resistance by targeting MAP2K6-p38 signalling in human colorectal adenocarcinoma cells. Nat Commun. 2016;7:12436. https://doi.org/10.1038/ncomms12436.

16. Due $H$, Schönherz $A A$, Ryø $L$, Primo MN, Jespersen DS, Thomsen EA, et al. MicroRNA-155 controls vincristine sensitivity and predicts superior clinical outcome in diffuse large B-cell lymphoma. Blood Adv. 2019;3:1185-96

17. Falgreen $\mathrm{S}$, et al. hemaClass. org: online one-by-one microarray normalization and classification of hematological cancers for precision medicine. PLoS One. 2016;11(10).

18. Falgreen S, Dybkær K, Young KH, Xu-Monette ZY, El-Galaly TC, Laursen MB, et al. Predicting response to multidrug regimens in cancer patients using cell line experiments and regularised regression models. BMC Cancer. 2015; 15:235. https://doi.org/10.1186/s12885-015-1237-6.

19. Lenz G, Wright G, Dave SS, Xiao W, Powell J, Zhao H, et al. Stromal gene signatures in large-B-cell lymphomas. N Engl J Med. 2008;359:2313-23. https://doi.org/10.1056/NEJMoa0802885.

20. Visco C, Li Y, Xu-Monette ZY, Miranda RN, Green TM, Li Y, et al. Comprehensive gene expression profiling and immunohistochemical studies support application of immunophenotypic algorithm for molecular subtype classification in diffuse large B-cell lymphoma: a report from the international DLBCL rituximab-CHOP Consortiu. Leukemia. 2012;26:2103-13.

21. Falgreen S, Laursen M, Bødker J, Kjeldsen M, Schmitz A, Nyegaard M, et al. Exposure time independent summary statistics for assessment of drug dependent cell line growth inhibition. BMC Bioinformatics. 2014;15:168. https://doi.org/10.1186/1471-2105-15-168.

22. Dybkær K, Bøgsted M, Falgreen S, Bødker JS, Kjeldsen MK, Schmitz A, et al. Diffuse large B-cell lymphoma classification system that associates normal B-cell subset phenotypes with prognosis. J Clin Oncol. 2015;33:1379-88. https://doi.org/10.1200/ JCO.2014.57.7080.

23. Brazma A, Hingamp P, Quackenbush J, Sherlock G, Spellman P, Stoeckert C, et al. Minimum information about a microarray experiment (MIAME) —-toward standards for microarray data. Nat Genet. 2001;29:365-71. https://doi.org/10. 1038/ng1201-365.

24. Irizarry RA, Hobbs B, Collin F, Beazer-Barclay YD, Antonellis KJ, Scherf U, et al. Exploration, normalization, and summaries of high density oligonucleotide array probe level data. Biostatistics. 2003;4:249-64. https://doi.org/10.1093/ biostatistics/4.2.249.

25. Gautier L, Cope L, Bolstad BM, Irizarry RA. Affy--analysis of Affymetrix GeneChip data at the probe level. Bioinformatics. 2004;20:307-15. https://doi.org/10.1093/ bioinformatics/btg405. 
26. Phipson B, Lee S, Majewski IJ, Alexander WS, Smyth GK. Robust hyperparameter estimation protects against hypervariable genes and improves power to detect differential expression. Ann Appl Stat. 2016;10:946-63.

27. Ritchie ME, Phipson B, Wu D, Hu Y, Law CW, Shi W, et al. Limma powers differential expression analyses for RNA-sequencing and microarray studies. Nucleic Acids Res. 2015;43:e47. https://doi.org/10. 1093/nar/gkv007.

28. Leek JT, Johnson WE, Parker HS, Fertig EJ, Jaffe AE, Storey JD, Zhang Y TL. sva: Surrogate Variable Analysis. R package version 3.28.0. 2018.

29. Ishwaran H, Kogalur UB, Blackstone EH, Lauer MS. Random survival forests. Ann Appl Stat. 2008;2:841-60 http://arxiv.org/abs/0811.1645v1.

30. Ishwaran H, Kogalur UB. Random survival forests for R. R News. 2007;7:25-31 https://cran.r-project.org/doc/Rnews/.

31. Ishwaran $H$, Kogalur UB. Fast unified random forests for survival, regression, and classification (RF-SRC). 2019. https://cran.r-project.org/package= randomForestSRC.

32. Cheson BD, Pfistner B, Juweid ME, Gascoyne RD, Specht L, Horning SJ, et al. Revised response criteria for malignant lymphoma. J Clin Oncol. 2007;25: 579-86. https://doi.org/10.1200/JCO.2006.09.2403.

33. Nakatani F, Ferracin M, Manara MC, Ventura S, Del Monaco V, Ferrari S, et al. miR-34a predicts survival of Ewing's sarcoma patients and directly influences cell chemo-sensitivity and malignancy. J Pathol J Pathol. 2012; 226:796-805. https://doi.org/10.1002/path.3007.

34. Pallasch CP, Leskov I, Braun CJ, Vorholt D, Drake A, Soto-Feliciano YM, et al. Sensitizing protective tumor microenvironments to antibody-mediated therapy. Cell. 2014;156:590-602. https://doi.org/10.1016/J.CELL.2013.12.041.

35. Wilson WH. Treatment strategies for aggressive lymphomas: what works? Hematol Am Soc Hematol Educ Progr. 2013;2013:584-90. https://doi.org/10. 1182/asheducation-2013.1.584.

36. Madsen ML, Due H, Ejskjær N, Jensen P, Madsen J, Dybkær K. Aspects of vincristine-induced neuropathy in hematologic malignancies: a systematic review. Cancer Chemother Pharmacol. 2019;84(3):471-85. https://doi.org/10. 1007/s00280-019-03884-5.

37. Lenz G, Wright GW, Tolga Emre NC, Kohlhammer H, Dave SS, Davis RE, et al. Molecular subtypes of diffuse large B-cell lymphoma arise by distinct genetic pathways. 2008. www.pnas.org/cgi/content/full/.

38. Pasqualucci L, Trifonov V, Fabbri G, Ma J, Rossi D, Chiarenza A, et al. Analysis of the coding genome of diffuse large B-cell lymphoma. Nat Genet. 2011; 43:830-7. https://doi.org/10.1038/ng.892.

39. Clark PM, Loher P, Quann K, Brody J, Londin ER, Rigoutsos I. Argonaute CLIP-Seq reveals miRNA targetome diversity across tissue types. Sci Rep. 2015;4:5947. https://doi.org/10.1038/srep05947.

40. Caramuta S, Lee L, Özata DM, Akçakaya P, Georgii-Hemming P, Xie H, et al. Role of microRNAs and microRNA machinery in the pathogenesis of diffuse large B-cell lymphoma. Blood Cancer J. 2013;3:e152. https://doi.org/10.1038/ bcj.2013.49.

41. Lawrie CH, Saunders NJ, Soneji S, Palazzo S, Dunlop HM, Cooper CDO, et al. MicroRNA expression in lymphocyte development and malignancy. Leukemia. 2008;22:1440-6. https://doi.org/10.1038/sj.leu.2405083.

42. Lawrie CH, Chi J, Taylor S, Tramonti D, Ballabio E, Palazzo S, et al. Expression of microRNAs in diffuse large $B$ cell lymphoma is associated with immunophenotype, survival and transformation from follicular lymphoma. J Cell Mol Med. 2009;13:1248-60. https://doi.org/10.1111/j.1582-4934.2008.00628.x.

43. Malumbres R, Sarosiek KA, Cubedo E, Ruiz JW, Jiang X, Gascoyne RD, et al. Differentiation stage-specific expression of microRNAs in B lymphocytes and diffuse large B-cell lymphomas. Blood. 2009;113:3754-64. https://doi.org/10. 1182/blood-2008-10-184077.

44. Lawrie CH, Soneji S, Marafioti T, Cooper CDO, Palazzo S, Paterson JC, et al. Microrna expression distinguishes between germinal center $B$ cell-like and activated B cell-like subtypes of diffuse large B cell lymphoma. Int J Cancer. 2007;121:1156-61. https://doi.org/10.1002/ijc.22800.

45. Chen W, Wang H, Chen H, Liu S, Lu H, Kong D, et al. Clinical significance and detection of microRNA-21 in serum of patients with diffuse large B-cell lymphoma in Chinese population. Eur J Haematol. 2014;92:407-12. https:// doi.org/10.1111/ejh.12263.

46. Zhong H, Xu L, Zhong J-H, Xiao F, Liu Q, Huang H-H, et al. Clinical and prognostic significance of miR-155 and miR-146a expression levels in formalinfixed/paraffin-embedded tissue of patients with diffuse large B-cell lymphoma. Exp Ther Med. 2012;3:763-70. https://doi.org/10.3892/etm.2012.502.

47. Li J, Smyth P, Flavin R, Cahill S, Denning K, Aherne S, et al. Comparison of miRNA expression patterns using total RNA extracted from matched samples of formalin-fixed paraffin-embedded (FFPE) cells and snap frozen cells. BMC Biotechnol. 2007;7:36. https://doi.org/10.1186/14726750-7-36.

48. Jones K, Nourse JP, Keane C, Bhatnagar A, Gandhi MK. Plasma microRNA are disease response biomarkers in classical Hodgkin lymphoma. Clin Cancer Res. 2014;20:253-64. https://doi.org/10.1158/1078-0432.CCR-13-1024.

49. Kurkewich JL, Hansen J, Klopfenstein N, Zhang H, Wood C, Boucher A, et al. The miR-23a 27a 24-2 microRNA cluster buffers transcription and signaling pathways during hematopoiesis. PLoS Genet. 2017;13:e1006887. https://doi. org/10.1371/journal.pgen.1006887.

50. Thai T-H, Calado DP, Casola S, Ansel KM, Xiao C, Xue Y, et al. Regulation of the germinal center response by microRNA-155. Science. 2007;316:604-8. https://doi.org/10.1126/science.1141229.

51. Qian B, Katsaros D, Lu L, Preti M, Durando A, Arisio R, et al. High miR-21 expression in breast cancer associated with poor disease-free survival in early stage disease and high TGF- $\beta 1$. Breast Cancer Res Treat. 2009;117:13140. https://doi.org/10.1007/s10549-008-0219-7.

52. Jiang S, Zhang H-W, Lu M-H, He X-H, Li Y, Gu H, et al. MicroRNA-155 functions as an OncomiR in breast cancer by targeting the suppressor of cytokine signaling 1 gene. Cancer Res. 2010;70:3119-27. https://doi.org/10. 1158/0008-5472.CAN-09-4250.

53. Yang CH, Yue J, Pfeffer SR, Fan M, Paulus E, Hosni-Ahmed A, et al. MicroRNA-21 promotes glioblastoma tumorigenesis by down-regulating insulin-like growth factor-binding protein-3 (IGFBP3). J Biol Chem. 2014;289: 25079-87. https://doi.org/10.1074/jbc.M114.593863.

54. Marsigliante S, D'Urso OF, Storelli C, Mallardo M, Gianfreda CD, Montinaro A, et al. miR-155 is up-regulated in primary and secondary glioblastoma and promotes tumour growth by inhibiting GABA receptors. Int J Oncol. 2012; 41:228-34. https://doi.org/10.3892/ijo.2012.1420.

55. Go H, Jang J-Y, Kim P-J, Kim Y-G, Nam SJ, Paik JH, et al. MicroRNA-21 plays an oncogenic role by targeting FOXO1 and activating the PI3KAKT pathway in diffuse large B-cell lymphoma. Oncotarget. 2015;6:15035-49. https://doi.org/10.18632/oncotarget.3729.

56. Babar IA, Cheng CJ, Booth CJ, Liang X, Weidhaas JB, Saltzman WM, et al Nanoparticle-based therapy in an in vivo microRNA-155 (miR-155)dependent mouse model of lymphoma. Proc Natl Acad Sci U S A. 2012;109: E1695-704. https://doi.org/10.1073/pnas.1201516109.

57. Medina PP, Nolde M, Slack FJ. OncomiR addiction in an in vivo model of microRNA-21-induced pre-B-cell lymphoma. Nature. 2010;467:86-90. https:// doi.org/10.1038/nature09284.

58. Bai H, Wei J, Deng C, Yang X, Wang C, Xu R. MicroRNA-21 regulates the sensitivity of diffuse large B-cell lymphoma cells to the CHOP chemotherapy regimen. Int J Hematol. 2013;97:223-31. https://doi.org/10.1007/s12185-012-1256-x.

59. Dybkær K, Due H, Brøndum RF, Young KH, Bøgsted M. Addition of DrugResponse Specific Micro-RNAs to the International Prognostic Index Improves Prognostic Stratification of GCB-DLBCL Patients Treated with RCHOP. Blood. 2019;134(Supplement_1):1623.

\section{Publisher's Note}

Springer Nature remains neutral with regard to jurisdictional claims in published maps and institutional affiliations.

Ready to submit your research? Choose BMC and benefit from:

- fast, convenient online submission

- thorough peer review by experienced researchers in your field

- rapid publication on acceptance

- support for research data, including large and complex data types

- gold Open Access which fosters wider collaboration and increased citations

- maximum visibility for your research: over $100 \mathrm{M}$ website views per year

At BMC, research is always in progress.

Learn more biomedcentral.com/submissions 